\title{
Weak Kaon Production off the Nucleon
}

\author{
M. Rafi Alam, ${ }^{1}$ I. Ruiz Simo, ${ }^{2}$ M. Sajjad Athar,${ }^{1}$ and M. J. Vicente Vacas $^{2}$ \\ ${ }^{1}$ Department of Physics, Aligarh Muslim University, Aligarh-202 002, India \\ ${ }^{2}$ Departamento de Física Teórica and IFIC, \\ Centro Mixto Universidad de Valencia-CSIC, \\ Institutos de Investigación de Paterna, E-46071 Valencia, Spain
}

\begin{abstract}
The weak kaon production off the nucleon induced by neutrinos is studied at the low and intermediate energies of interest for some ongoing and future neutrino oscillation experiments. This process is also potentially important for the analysis of proton decay experiments. We develop a microscopical model based on the SU(3) chiral Lagrangians. The basic parameters of the model are $f_{\pi}$, the pion decay constant, Cabibbo's angle, the proton and neutron magnetic moments and the axial vector coupling constants for the baryons octet, $D$ and $F$, that are obtained from the analysis of the semileptonic decays of neutron and hyperons. The studied mechanisms are the main source of kaon production for neutrino energies up to 1.2 to $1.5 \mathrm{GeV}$ for the various channels and the cross sections are large enough to be amenable to be measured by experiments such as Minerva and T2K.
\end{abstract}

PACS numbers: 25.30.Pt,13.15.+g,12.15.-y,12.39.Fe 


\section{INTRODUCTION}

With the recent developments of the atmospheric and accelerator based neutrino experiments it is now well known that neutrinos oscillate and have finite masses. Now, the main goal is to precisely determine the different parameters of the Pontecorvo-Maki-NakagawaSakata (PMNS) matrix, absolute neutrino masses, CP violating phase $\delta$, etc. The neutrino energy region of a few $\mathrm{GeV}$ is quite sensitive to the neutrino oscillation parameters. Therefore, most of the present experiments like MiniBooNE, K2K, T2K, No $\nu$ A, etc. have taken data or have been planned in this energy region. Neutrino detection proceeds basically through various channels of interaction with hadronic targets like quasielastic scattering, meson production, resonance excitations, etc... Therefore, a reliable estimate of these cross sections has become important. There is a considerable ongoing theoretical and experimental effort addressing this question (see e.g. the proceedings of the NUINT conference series [1] ) with many of the studies concentrated at low energies where quasielastic scattering and pion production dominate or in the deep inelastic scattering. However, in the discussed energy region, other not so well known processes like kaon and hyperon production may also become important. In principle, their cross sections are smaller than for the pionic processes because of phase space and the Cabibbo suppression for $\Delta S=1$ reactions. Nonetheless, in the coming years of precision neutrino physics, their knowledge could be relevant for the data analysis, apart from their own intrinsic interest related to the role played by the strange quarks in hadronic physics.

The currently available data is restricted to a few events measured in bubble chamber experiments [2 4]. However, this is expected to change soon. In particular, MINER $\nu \mathrm{A}$, a dedicated experiment to measure neutrino nucleus cross section using several nuclear targets like Carbon, Iron and Lead in the neutrino energy region of 1-20 GeV has recently started taking data. It is also planned to study specifically the strange particle production and it is expected that thousands of events would be accumulated where a kaon is produced in the final state [5].

On the theoretical side there are very few calculations which deal with strange particle production at low neutrino energies: single hyperon production [6, 7], the study of several kaon and hyperon production channels of Dewan [8] and the work of Shrock [9] who has analysed the $\Delta S=0$ processes. At higher energies, Amer has studied the strange particle 
production assuming the dominance of s-channel resonant mechanisms [10]. Also, in part as a consequence of the scarcity of theoretical work, the MonteCarlo generators used in the analysis of the experiments apply models that are not too well suited to describe the strangeness production at low energies. For instance, NEUT, used by Super-Kamiokande, K2K, SciBooNE and T2K, only considers associated kaon production implemented by a model based on the excitation and later decay of resonances [11]. A similar model is used by other event generators like NEUGEN [12], NUANCE [13] (see also discussion in Ref. [14]) and GENIE [15]. As it will be emphasized below, this approach is not appropriate for low energies strangeness production.

In neutrino induced reactions, the first inelastic reaction creating strange quarks is the single kaon production (without accompanying hyperons) ${ }^{1}$. This charged current (CC) $\Delta S=1$ process is particularly appealing for several reasons. One of them is the important background that could produce, due to atmospheric neutrino interactions, in the analysis of one of the main decay channels the proton has in many SUSY GUT models $\left(p \rightarrow \nu+K^{+}\right)$ [16 18]. A second reason is its simplicity from a theoretical point of view. At low energies, it is possible to obtain model independent predictions using Chiral Perturbation Theory $(\chi \mathrm{PT})$ and due to the absence of $S=1$ baryonic resonances, the range of validity of the calculation could be extended to higher energies than for other channels. Furthermore, the kaon associated production (with accompanying hyperons) has a higher energy threshold (1.10 vs. $0.79 \mathrm{GeV})$. This implies that even when the associated production is not Cabibbo suppressed, for a wide energy region (such as the ANL, the MiniBooNE or the T2K neutrino spectrum) single kaon production could still be dominant [8].

The paper is organized as follows. In Sec. II we present the formalism for CC single kaon production in neutrino nucleon scattering based on the Lagrangians of $\mathrm{SU}(3) \chi \mathrm{PT}$. We also discuss the differences with previous calculations. Results, discussions and our concluding remarks are presented in Sec. III.

\footnotetext{
${ }^{1}$ For antineutrinos the lowest threshold for $|\Delta S|=1$ reactions is much lower and corresponds to hyperon production.
} 


\section{FORMALISM}

The basic reaction for the neutrino induced charged current kaon production is

$$
\nu_{l}(k)+N(p) \rightarrow l\left(k^{\prime}\right)+N^{\prime}\left(p^{\prime}\right)+K\left(p_{k}\right)
$$

where $l=e, \mu$ and $N \& N^{\prime}=\mathrm{n}, \mathrm{p}$. The expression for the differential cross section in the laboratory (lab) frame for the above process is given by,

$$
d^{9} \sigma=\frac{1}{4 M E(2 \pi)^{5}} \frac{d \vec{k}^{\prime}}{\left(2 E_{l}\right)} \frac{d \vec{p}^{\prime}}{\left(2 E_{p}^{\prime}\right)} \frac{d \vec{p}_{k}}{\left(2 E_{K}\right)} \delta^{4}\left(k+p-k^{\prime}-p^{\prime}-p_{k}\right) \bar{\Sigma} \Sigma|\mathcal{M}|^{2},
$$

where $\vec{k}$ and $\overrightarrow{k^{\prime}}$ are the 3-momenta of the incoming and outgoing leptons in the lab frame with energy $E$ and $E^{\prime}$ respectively. The kaon lab momentum is $\vec{p}_{k}$ having energy $E_{K}$, $M$ is the nucleon mass, $\bar{\Sigma} \Sigma|\mathcal{M}|^{2}$ is the square of the transition amplitude matrix element averaged(summed) over the spins of the initial(final) state. At low energies, this amplitude can be written in the usual form as

$$
\mathcal{M}=\frac{G_{F}}{\sqrt{2}} j_{\mu}^{(L)} J^{\mu(H)}=\frac{g}{2 \sqrt{2}} j_{\mu}^{(L)} \frac{1}{M_{W}^{2}} \frac{g}{2 \sqrt{2}} J^{\mu(H)}
$$

where $j_{\mu}^{(L)}$ and $J^{\mu(H)}$ are the leptonic and hadronic currents respectively, $G_{F}=\sqrt{2} \frac{g^{2}}{8 M_{W}^{2}}=$ $1.16639(1) \times 10^{-5} \mathrm{GeV}^{-2}$ is the Fermi constant and $g$ is the gauge coupling. The leptonic current can be readily obtained from the standard model Lagrangian coupling the $W$ bosons to the leptons

$$
\mathcal{L}=-\frac{g}{2 \sqrt{2}}\left[W_{\mu}^{+} \bar{\nu}_{l} \gamma^{\mu}\left(1-\gamma_{5}\right) l+W_{\mu}^{-} \bar{l}^{\mu}\left(1-\gamma_{5}\right) \nu_{l}\right]=-\frac{g}{2 \sqrt{2}}\left[j_{(L)}^{\mu} W_{\mu}^{+}+h . c .\right]
$$

We consider four different channels that contribute to the hadronic current. They are depicted in Fig. 1. There is a contact term (CT), a kaon pole (KP) term, a u-channel process with a $\Sigma$ or $\Lambda$ hyperon in the intermediate state and finally a meson $(\pi, \eta)$ exchange term. For the specific reactions under consideration, there are not s-channel contributions given the absence of $S=1$ baryonic resonances. The current of the KP term is proportional to $q^{\mu}$. This implies, after contraction with the leptonic tensor, that the amplitude is proportional to the lepton mass and therefore very small.

The contribution of the different terms can be obtained in a systematic manner using $\chi \mathrm{PT}$. This allows to identify some terms that were missing in the approach of Ref. [8] which 

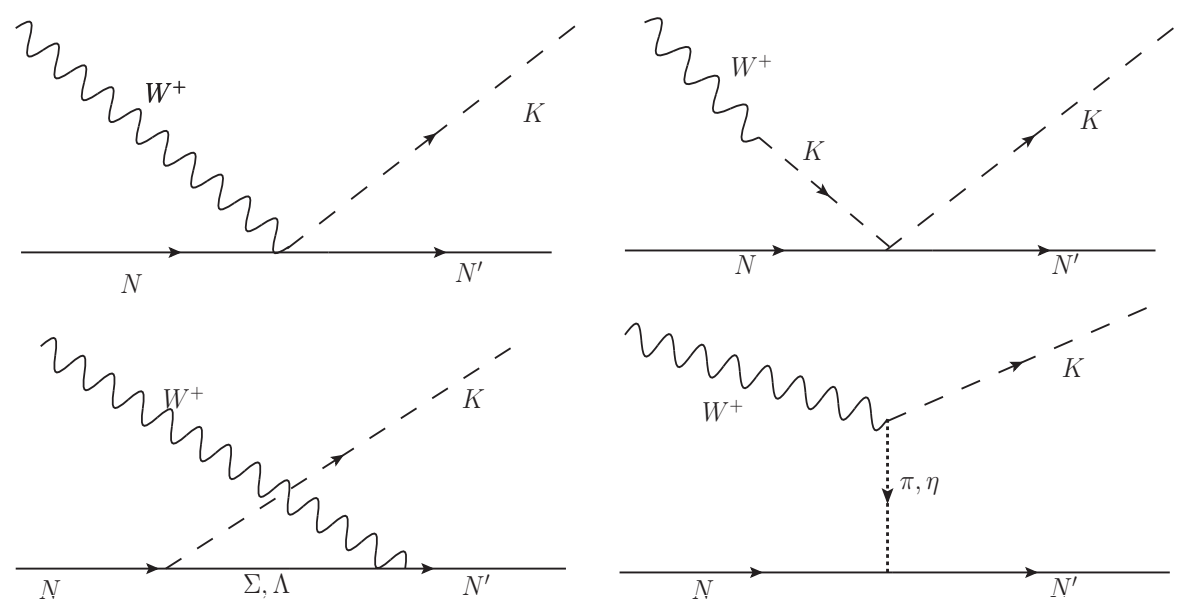

FIG. 1: Feynman diagrams for the process $\nu N \rightarrow l N^{\prime} K$. First row from left to right: contact term (labeled $\mathrm{CT}$ in the text), Kaon pole term (KP); second row: u-channel diagram $(C r \Sigma, C r \Lambda)$ and Pion(Eta) in flight $(\pi P,(\eta P)$

only included the u-channel diagrams in the calculation. The lowest-order $\mathrm{SU}(3)$ chiral Lagrangian describing the pseudoscalar mesons in the presence of an external current is

$$
\mathcal{L}_{M}^{(2)}=\frac{f_{\pi}^{2}}{4} \operatorname{Tr}\left[D_{\mu} U\left(D^{\mu} U\right)^{\dagger}\right]+\frac{f_{\pi}^{2}}{4} \operatorname{Tr}\left(\chi U^{\dagger}+U \chi^{\dagger}\right)
$$

where the parameter $f_{\pi}=92.4 \mathrm{MeV}$ is the pion decay constant, $U$ is the $\mathrm{SU}(3)$ representation of the meson fields

$$
\begin{aligned}
& U(x)=\exp \left(i \frac{\phi(x)}{f_{\pi}}\right), \\
& \phi(x)=\left(\begin{array}{ccc}
\pi^{0}+\frac{1}{\sqrt{3}} \eta & \sqrt{2} \pi^{+} & \sqrt{2} K^{+} \\
\sqrt{2} \pi^{-} & -\pi^{0}+\frac{1}{\sqrt{3}} \eta & \sqrt{2} K^{0} \\
\sqrt{2} K^{-} & \sqrt{2} \bar{K}^{0} & -\frac{2}{\sqrt{3}} \eta
\end{array}\right),
\end{aligned}
$$

and $D_{\mu} U$ is its covariant derivative

$$
D_{\mu} U \equiv \partial_{\mu} U-i r_{\mu} U+i U l_{\mu}
$$

Here, $l_{\mu}$ and $r_{\mu}$ correspond to left and right handed currents, that for the CC case are given by

$$
r_{\mu}=0, \quad l_{\mu}=-\frac{g}{\sqrt{2}}\left(W_{\mu}^{+} T_{+}+W_{\mu}^{-} T_{-}\right)
$$


with $W^{ \pm}$the $W$ boson fields and

$$
T_{+}=\left(\begin{array}{rrr}
0 & V_{u d} & V_{u s} \\
0 & 0 & 0 \\
0 & 0 & 0
\end{array}\right) ; \quad T_{-}=\left(\begin{array}{rrr}
0 & 0 & 0 \\
V_{u d} & 0 & 0 \\
V_{u s} & 0 & 0
\end{array}\right) .
$$

Here, $V_{i j}$ are the elements of the Cabibbo-Kobayashi-Maskawa matrix. The second term of the Lagrangian of Eq. 4, that incorporates the explicit breaking of chiral symmetry coming from the quark masses [19], is not relevant for our study.

The lowest-order chiral Lagrangian for the baryon octet in the presence of an external current can be written in terms of the $\mathrm{SU}(3)$ matrix

$$
B=\left(\begin{array}{ccc}
\frac{1}{\sqrt{2}} \Sigma^{0}+\frac{1}{\sqrt{6}} \Lambda & \Sigma^{+} & p \\
\Sigma^{-} & -\frac{1}{\sqrt{2}} \Sigma^{0}+\frac{1}{\sqrt{6}} \Lambda & n \\
\Xi^{-} & \Xi^{0} & -\frac{2}{\sqrt{6}} \Lambda
\end{array}\right)
$$

as

$$
\mathcal{L}_{M B}^{(1)}=\operatorname{Tr}[\bar{B}(i \not D-M) B]-\frac{D}{2} \operatorname{Tr}\left(\bar{B} \gamma^{\mu} \gamma_{5}\left\{u_{\mu}, B\right\}\right)-\frac{F}{2} \operatorname{Tr}\left(\bar{B} \gamma^{\mu} \gamma_{5}\left[u_{\mu}, B\right]\right)
$$

where $M$ denotes the mass of the baryon octet, and the parameters $D=0.804$ and $F=0.463$ can be determined from the baryon semileptonic decays [20]. The covariant derivative of $B$ is given by

$$
D_{\mu} B=\partial_{\mu} B+\left[\Gamma_{\mu}, B\right]
$$

with

$$
\Gamma_{\mu}=\frac{1}{2}\left[u^{\dagger}\left(\partial_{\mu}-i r_{\mu}\right) u+u\left(\partial_{\mu}-i l_{\mu}\right) u^{\dagger}\right]
$$

where we have introduced $u^{2}=U$. Finally,

$$
u_{\mu}=i\left[u^{\dagger}\left(\partial_{\mu}-i r_{\mu}\right) u-u\left(\partial_{\mu}-i l_{\mu}\right) u^{\dagger}\right]
$$

The next order meson baryon Lagrangian contains many new terms (see for instance Ref. [21]). Their importance for kaon production will be small at low energies and there are some uncertainties in the coupling constants. Nonetheless, for consistency with previous calculations, we will include the contribution to the weak magnetism coming from the pieces

$$
\mathcal{L}_{M B}^{(2)}=d_{5} \operatorname{Tr}\left(\bar{B}\left[f_{\mu \nu}^{+}, \sigma^{\mu \nu} B\right]\right)+d_{4} \operatorname{Tr}\left(\bar{B}\left\{f_{\mu \nu}^{+}, \sigma^{\mu \nu} B\right\}\right)+\ldots
$$

where the tensor $f_{\mu \nu}^{+}$can be reduced for our study to

$$
f_{\mu \nu}^{+}=\partial_{\mu} l_{\nu}-\partial_{\nu} l_{\mu}-i\left[l_{\mu}, l_{\nu}\right]
$$


TABLE I: Values of the parameters appearing in the hadronic currents.

\begin{tabular}{|c|ccccccc|}
\hline \hline Process & $A_{C T}$ & $B_{C T}$ & $A_{C r \Sigma}$ & $A_{C r \Lambda}$ & $A_{K P}$ & $A_{\pi P}$ & $A_{\eta P}$ \\
\hline \hline$\nu n \rightarrow l K n$ & 1 & $\mathrm{D}-\mathrm{F}$ & $-(\mathrm{D}-\mathrm{F})$ & 0 & 1 & 1 & 1 \\
\hline$\nu p \rightarrow l K p$ & 2 & $-\mathrm{F}$ & $-(\mathrm{D}-\mathrm{F}) / 2$ & $(\mathrm{D}+3 \mathrm{~F})$ & 2 & -1 & 1 \\
\hline$\nu n \rightarrow l K p$ & 1 & $-\mathrm{D}-\mathrm{F}$ & $(\mathrm{D}-\mathrm{F}) / 2$ & $(\mathrm{D}+3 \mathrm{~F})$ & 1 & -2 & 0 \\
\hline \hline
\end{tabular}

In this case, the coupling constants are fully determined by the proton and neutron anomalous magnetic moments. This same approximation has also been used in calculations of single pion production induced by neutrinos [22]. Now, writing the amplitude for the coupling of the $W$ boson to the hadrons for each of the terms in the form $\frac{g}{2 \sqrt{2}}\left(J_{H}^{\mu} W_{\mu}^{+}+\right.$h.c. $)$, for consistency with Eq. 2, we get the following contributions to the hadronic current

$$
\begin{aligned}
\left.j^{\mu}\right|_{C T}= & -i A_{C T} V_{u s} \frac{\sqrt{2}}{2 f_{\pi}} \bar{N}\left(p^{\prime}\right)\left(\gamma^{\mu}+\gamma^{\mu} \gamma^{5} B_{C T}\right) N(p), \\
\left.j^{\mu}\right|_{C r \Sigma}= & i A_{C r \Sigma} V_{u s} \frac{\sqrt{2}}{2 f_{\pi}} \bar{N}\left(p^{\prime}\right)\left(\gamma^{\mu}+i \frac{\mu_{p}+2 \mu_{n}}{2 M} \sigma^{\mu \nu} q_{\nu}+(D-F)\left(\gamma^{\mu}-\frac{q^{\mu}}{q^{2}-M_{k}^{2}} \not\right) \gamma^{5}\right) \\
& \times \frac{\not p-\not p_{k}+M_{\Sigma}}{\left(p-p_{k}\right)^{2}-M_{\Sigma}^{2}} \not p_{k} \gamma^{5} N(p), \\
\left.j^{\mu}\right|_{C r \Lambda}= & i A_{C r \Lambda} V_{u s} \frac{\sqrt{2}}{4 f_{\pi}} \bar{N}\left(p^{\prime}\right)\left(\gamma^{\mu}+i \frac{\mu_{p}}{2 M} \sigma^{\mu \nu} q_{\nu}-\frac{D+3 F}{3}\left(\gamma^{\mu}-\frac{q^{\mu}}{q^{2}-M_{k}^{2}} \not q\right) \gamma^{5}\right) \\
& \times \frac{\not p-p_{k}+M_{\Lambda}}{\left(p-p_{k}\right)^{2}-M_{\Lambda}^{2}} \not p_{k} \gamma^{5} N(p), \\
\left.j^{\mu}\right|_{K P}= & i A_{K P} V_{u s} \frac{\sqrt{2}}{4 f_{\pi}} \bar{N}\left(p^{\prime}\right)\left(\not q+\not p_{k}\right) N(p) \frac{1}{q^{2}-M_{k}^{2}} q^{\mu}, \\
\left.j^{\mu}\right|_{\pi}= & i A_{\pi P} V_{u s}(D+F) \frac{\sqrt{2}}{2 f_{\pi}} \frac{M}{\left(q-p_{k}\right)^{2}-M_{\pi}^{2}} \bar{N}\left(p^{\prime}\right) \gamma^{5} \cdot\left(q^{\mu}-2 p_{k}{ }^{\mu}\right) N(p), \\
\left.j^{\mu}\right|_{\eta}= & i A_{\eta P} V_{u s}(D-3 F) \frac{\sqrt{2}}{2 f_{\pi}} \frac{M}{\left(q-p_{k}\right)^{2}-M_{\eta}^{2}} \bar{N}\left(p^{\prime}\right) \gamma^{5} \cdot\left(q^{\mu}-2 p_{k}{ }^{\mu}\right) N(p),
\end{aligned}
$$

where, $q=k-k^{\prime}$ is the four momentum transfer, $V_{u s}=\sin \theta=0.22$ where $\theta$ is the Cabibbo angle, $N(\cdot), \bar{N}(\cdot)$ denote the nucleon spinors, $\mu_{p}=1.7928$ and $\mu_{n}=-1.9130$ are the proton and neutron anomalous magnetic moments. The value of the various parameters of the formulas are shown in Table I. One can notice the induced pseudoscalar form factor in the $\left.j^{\mu}\right|_{C r \Sigma, C r \Lambda}$ currents, which takes into account the coupling of the $W$ boson to the baryon through a kaon. However, as for the KP term, its contribution is suppressed by a factor proportional to the final lepton mass and is negligible. Now, we discuss in some detail the 
terms that appear in the coupling of the weak currents to the octet baryons in the u-channel diagrams. With very general symmetry arguments, this coupling can be described in terms of three vector and three axial form factors. Following the notation of Ref. [20] we have

$$
\begin{aligned}
O_{V}^{\mu} & =f_{1} \gamma^{\mu}+\frac{f_{2}}{M_{B}} \sigma^{\mu \nu} q_{\nu}+\frac{f_{3}}{M_{B}} q^{\mu}, \\
O_{A}^{\mu} & =\left(g_{1} \gamma^{\mu}+\frac{g_{2}}{M_{B}} \sigma^{\mu \nu} q_{\nu}+\frac{g_{3}}{M_{B}} q^{\mu}\right) \gamma^{5},
\end{aligned}
$$

where $M_{B}$ is the baryon mass. At the order considered, the chiral Lagrangian provides finite values for $f_{1}$, the weak magnetism form factor $f_{2}, g_{1}$ and a pole contribution to $g_{3}$. The scalar $f_{3}$ and a non-pole part of the pseudoscalar $g_{3}$ form factors would only appear at higher orders of the chiral expansion. Furthermore, their contribution to the amplitude is suppressed by a $m_{l}$ (lepton mass) factor and they are usually neglected. The value of $g_{2}$ vanishes in the limit of exact $\mathrm{SU}(3)$ symmetry and there is very little experimental information about it. In fact, it is also neglected in most analyses of hyperon phenomenology [23]. The values of $f_{1}$ and $g_{1}$ obtained from the lowest order chiral Lagrangians describe well the hyperon semileptonic decays $[20,23,24]$.

Eventually, if the cross sections for the discussed processes were measured with some precision, one could use them to explore these form factors at several $q^{2}$ values. The current experimental information, based on the semileptonic decays, covers only a very reduced range for this magnitude.

Finally, we consider the $q^{2}$ dependence of the weak current couplings provided by the chiral Lagrangians discussed earlier. We should remark that, even at relatively low energies and low momenta of the hadrons involved in our study, $q^{2}$ reaches moderate values. The $q^{2}$ dependences of the needed form factors (e.g. K $\pi, \mathrm{YN}$ ) are poorly known if at all. Several prescriptions have been used in the literature. For instance, for quasielastic scattering and single pion production, the vector form factors are usually related to the well known nucleon electromagnetic ones (see e.g. [22, 25, 26] and references therein). This procedure is well suited for these two cases because of isospin symmetry. However, in the $\mathrm{SU}(3)$ sector we expect to have some symmetry breaking effects. Similarly, for the axial form factors, a $q^{2}$ dependence obtained from the nucleon-nucleon transition obtained in neutrino nucleon quasielastic scattering is normally used. However, the axial mass is not well established and it runs from values around $1 \mathrm{GeV}[27,28]$ to $1.2 \mathrm{GeV}$ recently obtained by the $\mathrm{K} 2 \mathrm{~K}[29]$ and MiniBooNE [30] collaborations. Again here, we expect a different behavior for the hyperon- 


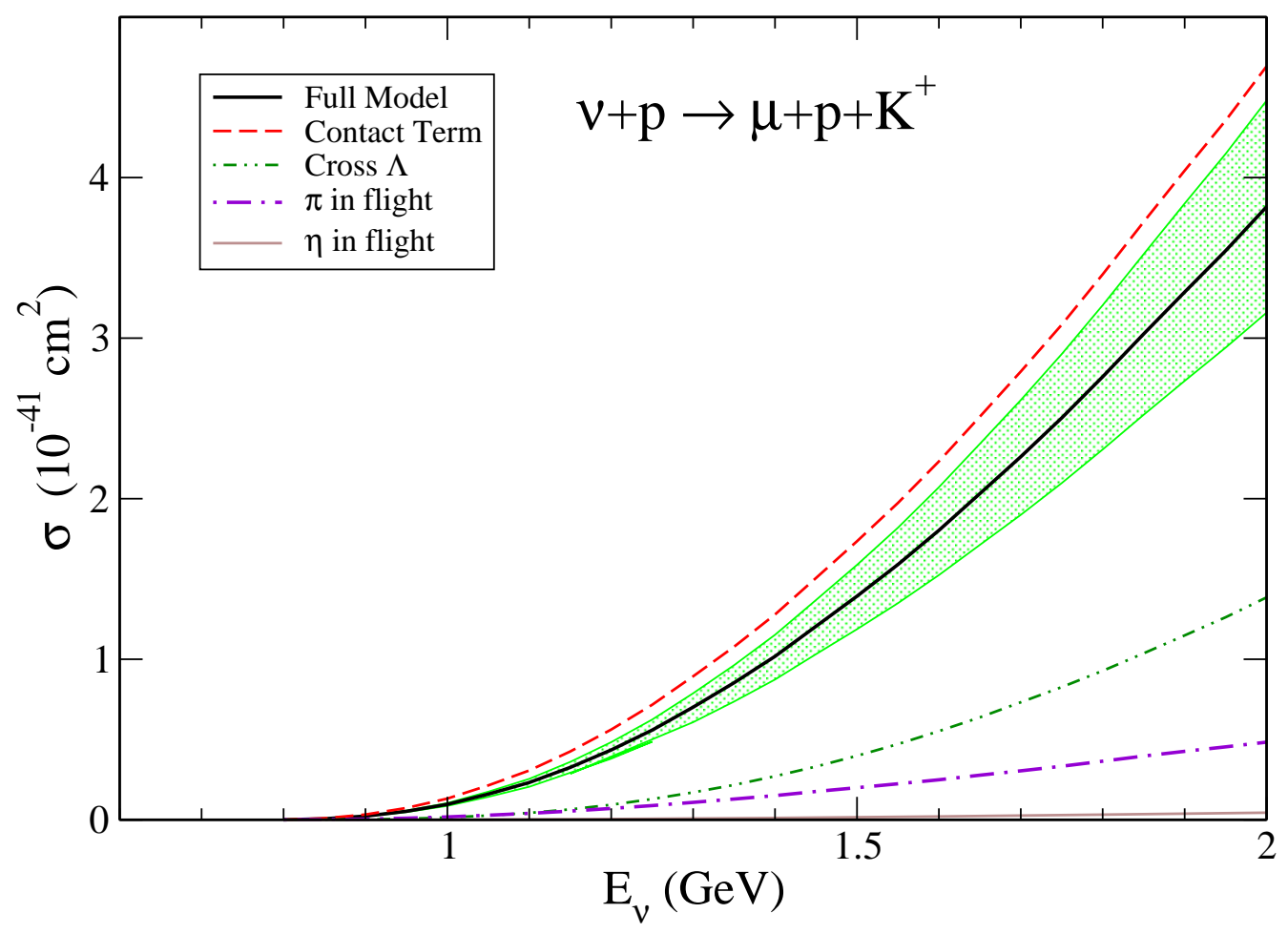

FIG. 2: Contribution of the different terms to the total cross section for the $\nu_{\mu} p \rightarrow \mu K^{+} p$ reaction.

nucleon vertices. One of the possible choices (e.g. [10]) is to use a dipole form with the mass of the vector(axial) meson that could couple the baryon to the current. In this work, in view of the present uncertainties, we adopt a global dipole form factor $F\left(q^{2}\right)=1 /\left(1-q^{2} / M_{F}^{2}\right)^{2}$, with a mass $M_{F} \simeq 1 \mathrm{GeV}$ that multiplies the hadronic currents. Its effect, that should be small at low neutrino energies will give an idea of the uncertainties of the calculation and will be explored in the next section.

\section{RESULTS AND DISCUSSION}

We consider the following reactions:

$$
\begin{aligned}
& \nu_{l}+p \rightarrow l^{-}+K^{+}+p \quad(l=e, \mu) \\
& \nu_{l}+n \rightarrow l^{-}+K^{0}+p \\
& \nu_{l}+n \rightarrow l^{-}+K^{+}+n
\end{aligned}
$$

The total scattering cross section $\sigma$ has been obtained by using Eq. (2) after integrating over the kinematical variables. In Figs. (2,4), we present the results of the contributions of 


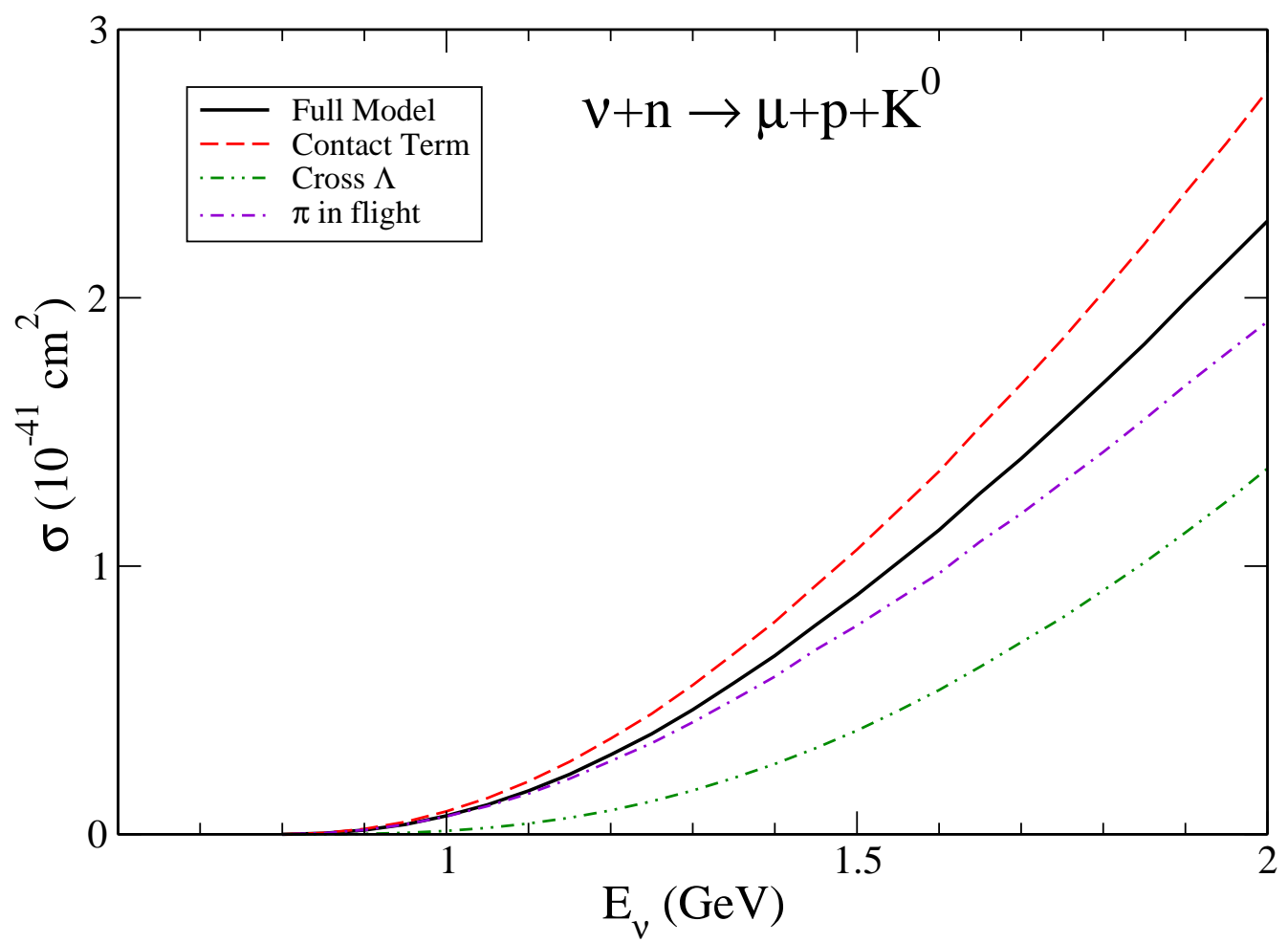

FIG. 3: Contribution of the different terms to the total cross section for the $\nu_{\mu} n \rightarrow \mu K^{0} p$ reaction.

the different diagrams to the total cross sections. The kaon pole contributions are negligible at the studied energies and are not shown in the figures although they are included in the full model curves. We observe the relevance of the contact term, not included in previous calculations. Starting from the $\nu_{\mu}+p \rightarrow \mu^{-}+K^{+}+p$ channel, we find that the contact term is in fact dominant, followed by the u-channel diagram with a $\Lambda$ intermediate state and the $\pi$ exchange term. As observed by Dewan [8] the u-channel $\Sigma$ contribution is much less important, basically because of the larger coupling $(N K \Lambda \gg N K \Sigma)$ of the strong vertex. The curve labeled as Full Model has been calculated with a dipole form factor with a mass of $1 \mathrm{GeV}$. The band corresponds to changing up and down this mass by a 10 percent. A similar effect is found in the other channels and we will only show the results for the central value of $1 \mathrm{GeV}$. We have also checked that the cross section obtained without the contact term and after correcting for the different values of the Cabibbo angle and the Yukawa strong coupling agrees well with the result of Fig. 7 of Ref. [8] at its lowest energy. Higher energies are well beyond the scope of our model.

The process $\nu_{\mu}+n \rightarrow \mu^{-}+K^{0}+p$ has a cross section of a similar size and the contact 


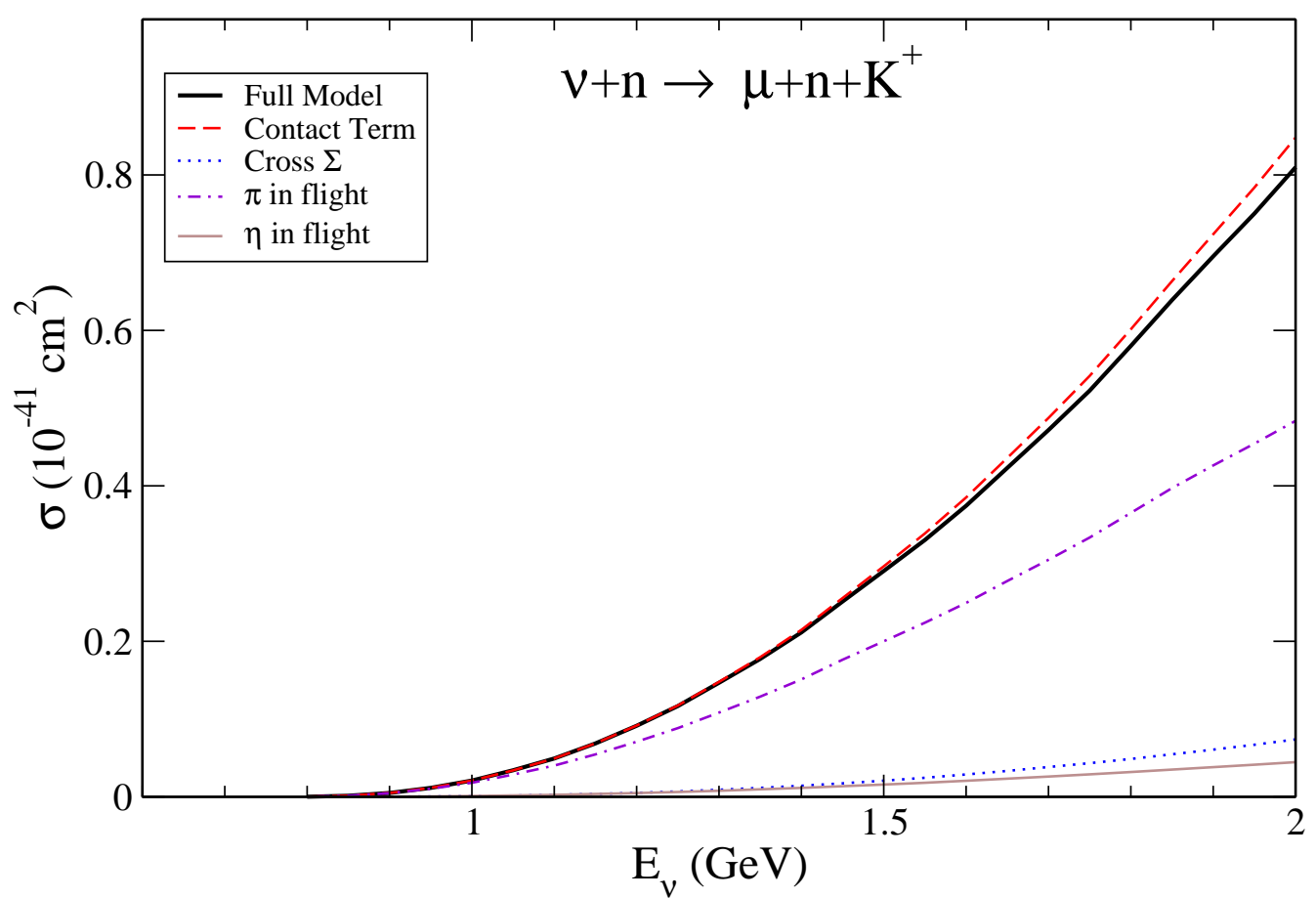

FIG. 4: Contribution of the different terms to the total cross section for the $\nu_{\mu} n \rightarrow \mu K^{+} n$ reaction.

term is also the largest one, followed by the $\pi$ exchange diagram and the $\mathrm{u}$-channel $(\Lambda)$ term. The rate of growth of the latter is somehow larger and could become more important at higher energies. As for the previous channel, we observe a destructive interference between the different terms and the cross section obtained with the full model is smaller than that produced by the contact term alone.

Finally, the reaction $\nu_{l}+n \rightarrow l^{-}+K^{+}+n$ has a smaller cross section. The pion exchange term is substantially bigger than the u-channel mechanisms, as already noted in Ref. [8]. The contact term is also dominant for this channel and the total cross section calculated only with this term practically coincides with the full result. Therefore, we have found that the contact terms, required by symmetry, play a major role in the description of the kaon production induced by neutrinos at low energies.

Above the energy threshold for the production of kaons accompanied by hyperons, this latter kind of processes could have larger cross sections due to the larger coupling for $\Delta S=0$, $\left(V_{u d}\right.$ vs $\left.V_{u s}\right)$. To explore this question and the range of energies where the processes we have studied are relevant we compare our results in Fig. 5, with the values for the associated production obtained by means of the GENIE Monte Carlo program [15]. We observe that, 


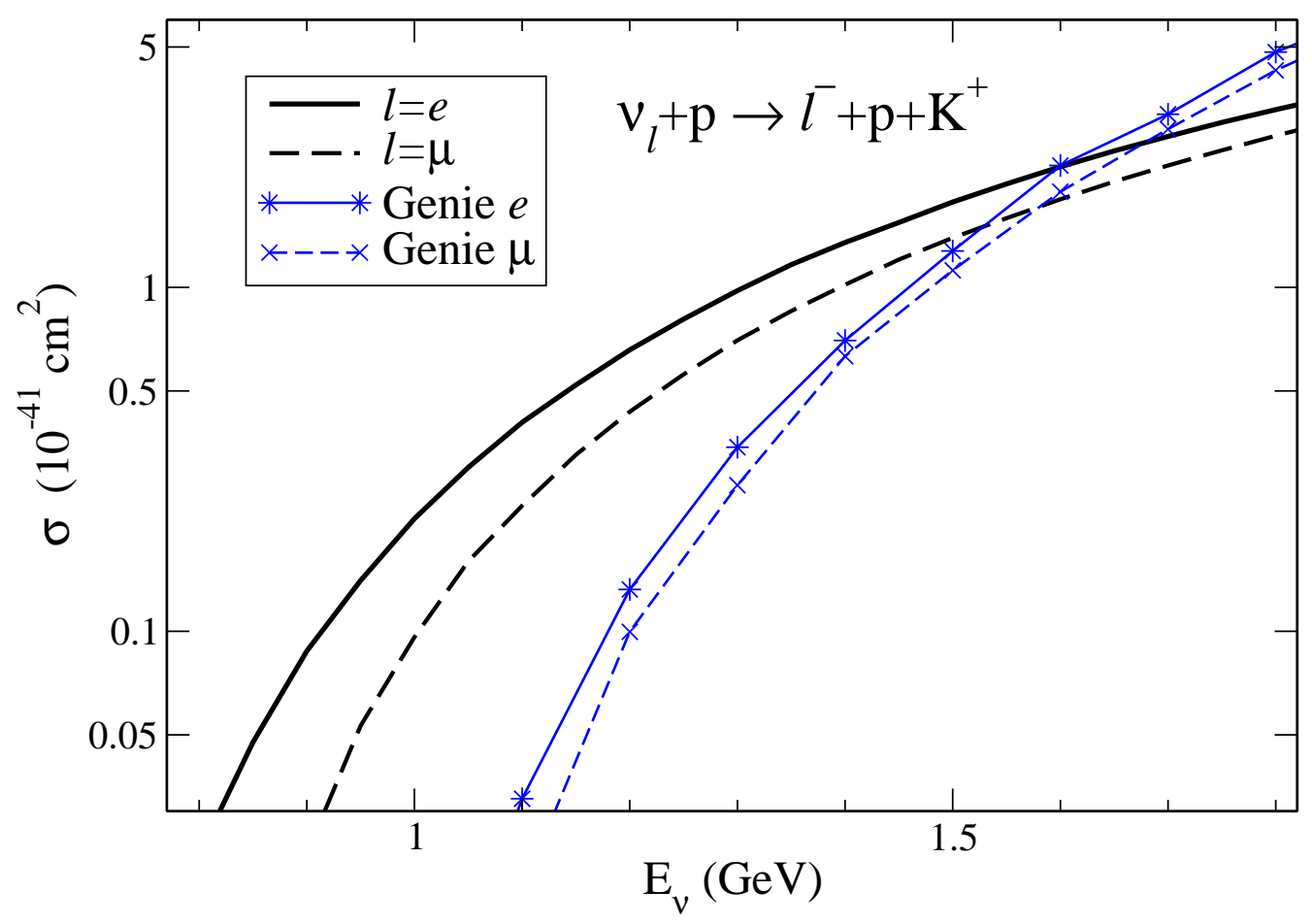

FIG. 5: Cross sections as a function of the neutrino energy for single kaon production vs. associated production obtained with Genie [15].

due to the difference between the energy thresholds, single kaon production for the $\nu_{l}+p \rightarrow$ $l^{-}+K^{+}+p$ is clearly dominant for neutrinos of energies below $1.5 \mathrm{GeV}$. For the other two channels associated production becomes comparable at lower energies. Still, single $K^{0}$ production off neutrons is larger than the associated production up to $1.3 \mathrm{GeV}$ and even the much smaller $K^{+}$production off neutrons is larger than the associated production up to 1.1 $\mathrm{GeV}$. The consideration of these $\Delta S=1$ channels is therefore important for the description of strangeness production for all low energy neutrino spectra and should be incorporated in the experimental analysis.

In Table [I] we show the total cross section results for the three channels averaged over the ANL [31], the MiniBooNE [32] and the off-axis (2.5 degrees) T2K [33] muon neutrino fluxes, all of them peaking at around $0.6 \mathrm{GeV}$. After normalization of the neutrino flux $\phi$ we have

$$
\bar{\sigma}=\int_{E_{\mathrm{th}}}^{E_{\mathrm{high}}} d E \phi(E) \sigma(E),
$$

where $E_{\text {th }}$ is the threshold energy for each process and $E_{\text {high }}$ is the maximum neutrino 
TABLE II: Cross sections averaged over the neutrino flux at different laboratories in units of $10^{-41}$ $\mathrm{cm}^{2}$. Theoretical uncertainties correspond to a $10 \%$ variation of the form factor mass.

\begin{tabular}{|c|ccc|}
\hline \hline Process & ANL & MiniBooNE & T2K \\
\hline \hline$\nu_{\mu} n \rightarrow \mu^{-} K^{+} n$ & $0.06(1)$ & $0.07(1)$ & $0.09(1)$ \\
\hline$\nu_{\mu} p \rightarrow \mu^{-} K^{+} p$ & $0.28(5)$ & $0.32(5)$ & $0.43(8)$ \\
\hline$\nu_{\mu} n \rightarrow \mu^{-} K^{0} p$ & $0.17(3)$ & $0.20(3)$ & $0.25(5)$ \\
\hline \hline
\end{tabular}

TABLE III: Number of events calculated for single kaon production in water corresponding to the SuperK analysis for atmospheric neutrinos.

\begin{tabular}{|c|cc|}
\hline \hline Process & Events $e^{-}$ & Events $\mu^{-}$ \\
\hline$\nu_{l} n \rightarrow l^{-} n K^{+}$ & 0.16 & 0.27 \\
$\nu_{l} n \rightarrow l^{-} p K^{0}$ & 0.45 & 0.73 \\
$\nu_{l} p \rightarrow l^{-} p K^{+}$ & 0.95 & 1.55 \\
\hline Total & 1.56 & 2.55 \\
\hline \hline
\end{tabular}

energy. As discussed previously, in these three cases, the neutrino energies are low enough for single kaon production to be relevant as compared to associated kaon production. Also the invariant mass of the hadronic system and the transferred momentum only reach the relatively small values where our model is more reliable.

We can get an idea of the magnitude of these channels by comparing their cross section to some recent results. For instance, the cross section for neutral current $\pi^{0}$ production per nucleon has been measured by the MiniBooNE collaboration [34] obtaining $\bar{\sigma}=(4.76 \pm 0.05 \pm$ $0.76) \times 10^{-40} \mathrm{~cm}^{2}$ with a data set of some twenty thousand valid events. The cross sections predicted by our model with the same neutrino flux are around two orders of magnitude smaller, what means that a few hundreds of kaons should have been produced.

The atmospheric spectrum [35] also peaks at very low energies and our model should be very well suited to analyse the kaon production. In Table III, we show the number of kaon events that we obtain for the 22.5 kTons of a water target and a period of 1489 days as in the SuperK analysis [17, 36] of proton decay. As in the quoted paper, we include cuts in the electron momentum $\left(p_{e}>100 \mathrm{MeV}\right)$ and muon momentum $\left(p_{e}>200 \mathrm{MeV}\right)$. We find 
that single kaon production is a very small source of background. In the SuperK analysis the kaon production was modeled following Ref. [37, 38] and only included associated kaon production. Although some of the cuts applied in their analysis, such as looking for an accompanying hyperon, are useless for our case, we find that this source of background is negligible, given the smallness of our results and the totally different energy distribution of kaons and final leptons in the production and decay reactions.

Finally, we study the values of $Q^{2}$ involved in the reaction for the typical neutrino energies we have considered. If high values of this magnitude are relevant, the results would be sensitive to higher orders of the chiral Lagrangians and/or a more precise description of the form factors. We show the $Q^{2}$ distribution in Fig. (6) for the three studied channels at a neutrino energy $\mathrm{E}_{\nu}=1 \mathrm{GeV}$. The reactions are always forward peaked (for the final lepton), even in the absence of any form factor $\left(F\left(q^{2}\right)=1\right.$ ), favouring relatively small values of the momentum transfer. In this figure, we also show the dependence of the cross section on the mass of the final lepton that reduces the cross section at low $Q^{2}$ values. The process $\nu_{e}+n \rightarrow e^{-}+K^{0}+p$ shows a slightly different behavior that reflects an important (and $Q^{2}$ dependent) interference between the pion exchange and the contact terms.

Till now we have discussed the kaon production off free nucleons. However, most of the experiments are carried out on detectors containing complex nuclei such as iron, oxygen or carbon. On the other hand, nuclear effects are known to be quite large for pion production induced by neutrinos [26, 39 41]. Fortunately, this question is much simpler for the kaons. First, because there is no kaon absorption and the final state interaction is reduced to a repulsive potential, small when compared with the typical kaon energies. Second, because of the absence of resonant channels in the production processes. We could remember here that some of the major nuclear effects for pion production are originated by the modification of the $\Delta(1232)$ properties on nuclei. Other nuclear effects, such as Fermi motion and Pauli blocking will only produce minor changes on the cross section and can easily be implemented in the Monte Carlo codes.

In summary, we have developed a microscopical model for single kaon production off nucleons induced by neutrinos based on the SU(3) chiral Lagrangians. This model should be quite reliable at low and intermediate energies given the absence of $S=1$ baryonic resonances in the s-channel. The parameters of the model are well known: $f_{\pi}$, the pion decay constant, Cabibbo's angle, the proton and neutron magnetic moments and the axial 


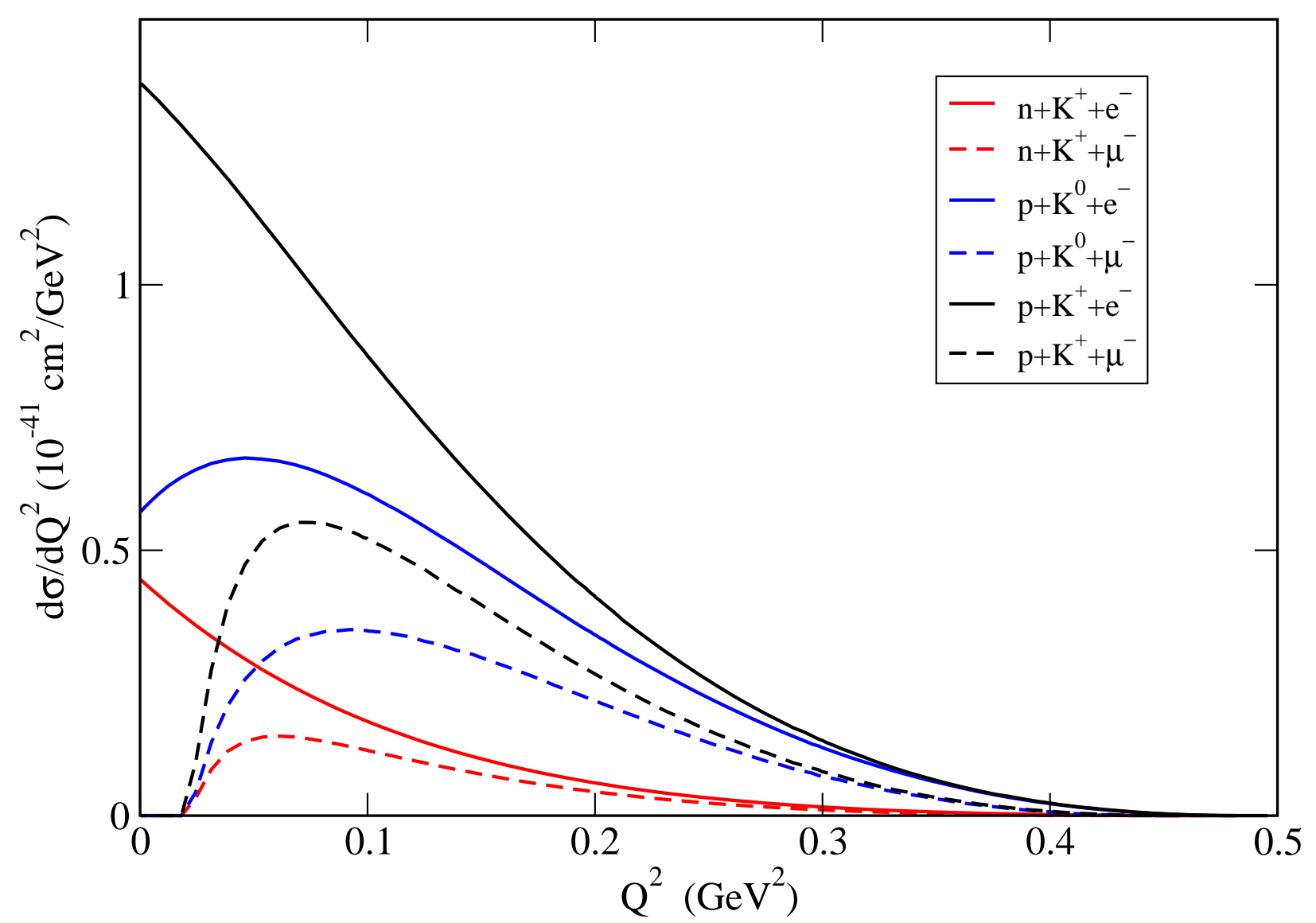

FIG. 6: $\frac{d \sigma}{d Q^{2}}$ at $E_{\nu}=1 G e V$ for single kaon production induced by neutrinos. The curves are labeled according to the final state of the process.

vector coupling constants for the baryons octet, $D$ and $F$. For the latter ones, we have taken the values obtained from the analysis of the hyperon semileptonic decays. The importance of higher order terms has been estimated using a dipole form factor with a mass around $1 \mathrm{GeV}$ and exploring the dependence of our results on this parameter.

We obtain cross sections that are around two orders of magnitude smaller than for pion production for neutrino spectra such as those of ANL or MiniBooNE. This can be understood because of the Cabibbo suppression and of the smaller phase space. Nonetheless, the cross sections are large enough to be measured, for instance, with the expected Minerva and T2K fluxes and could have been well measured at MiniBooNE. We have also found, that due to the higher threshold of the associated kaon production, the reactions we have studied are the dominant source of kaons for a wide range of energies, and thus their study is important for some low energy experiments and for the atmospheric neutrino flux. 


\section{Acknowledgments}

We want to acknowledge discussions with P. Stamoulis. This work is partly supported by DGICYT Contract No. FIS 2006-03438, the Generalitat Valenciana in the program Prometeo and the EU Integrated Infrastructure Initiative Hadron Physics Project under contract RII3-CT-2004-506078. I.R.S. acknowledges support from the Ministerio de Educación.M.R.A. wishes to acknowledge the financial support from the University of Valencia and Aligarh Muslim University under the academic exchange program and also to the DST, Government of India for the financial support under the grant SR/S2/HEP-0001/2008.

[1] Proc. of "SIXTH INTERNATIONAL WORKSHOP ON NEUTRINO-NUCLEUS INTERACTIONS IN THE FEW-GEV REGION (NUINT-09)", AIP Conf. Proc. 1189 (2009).

[2] S. J. Barish et al., Phys. Rev. Lett. 33, 1446 (1974).

[3] S. J. Barish et al., Phys. Rev. D 19 (1979) 2521.

[4] N. J. Baker et al., Phys. Rev. D 24, 2779 (1981).

[5] N. Solomey [Minerva Collaboration], Nucl. Phys. Proc. Suppl. 142, 74 (2005).

[6] S. K. Singh and M. J. Vicente Vacas, Phys. Rev. D 74, 053009 (2006).

[7] S. L. Mintz and L. Wen, Eur. Phys. J. A 33 (2007) 299.

[8] H. K. Dewan, Phys. Rev. D 24, 2369 (1981).

[9] R. E. Shrock, Phys. Rev. D 12, 2049 (1975).

[10] A. A. Amer, Phys. Rev. D 18, 2290 (1978).

[11] Y. Hayato, Acta Phys. Polon. B 40 (2009) 2477.

[12] H. Gallagher, Nucl. Phys. Proc. Suppl. 112 (2002) 188.

[13] D. Casper, Nucl. Phys. Proc. Suppl. 112 (2002) 161.

[14] G. P. Zeller, arXiv:hep-ex/0312061.

[15] C. Andreopoulos et al., Nucl. Instrum. Meth. A 614 (2010) 87.

[16] T. Marrodan Undagoitia et al., J. Phys. Conf. Ser. 39, 269 (2006).

[17] K. Kobayashi et al. [Super-Kamiokande Collaboration], Phys. Rev. D 72, 052007 (2005).

[18] W. A. Mann, T. Kafka, M. Derrick, B. Musgrave, R. Ammar, D. Day and J. Gress, Phys. Rev. D 34, 2545 (1986). 
[19] S. Scherer, Adv. Nucl. Phys. 27, 277 (2003).

[20] N. Cabibbo, E. C. Swallow and R. Winston, Ann. Rev. Nucl. Part. Sci. 53, 39 (2003).

[21] J. A. Oller, M. Verbeni and J. Prades, JHEP 0609 (2006) 079.

[22] E. Hernandez, J. Nieves and M. Valverde, Phys. Rev. D 76, 033005 (2007).

[23] R. Flores-Mendieta, Phys. Rev. D 70 (2004) 114036.

[24] L. S. Geng, J. Martin Camalich and M. J. Vicente Vacas, Phys. Rev. D 79 (2009) 094022.

[25] O. Benhar, N. Farina, H. Nakamura, M. Sakuda and R. Seki, Phys. Rev. D 72, 053005 (2005).

[26] T. Leitner, O. Buss, U. Mosel and L. Alvarez-Ruso, Phys. Rev. C 79 (2009) 038501.

[27] V. Bernard, L. Elouadrhiri and U. G. Meissner, J. Phys. G 28 (2002) R1.

[28] K. S. Kuzmin, V. V. Lyubushkin and V. A. Naumov, Eur. Phys. J. C 54 (2008) 517.

[29] R. Gran et al. [K2K Collaboration], Phys. Rev. D 74 (2006) 052002.

[30] A. A. Aguilar-Arevalo et al. [MiniBooNE Collaboration], Phys. Rev. Lett. 100 (2008) 032301.

[31] S. J. Barish et al., Phys. Rev. D 16 (1977) 3103.

[32] A. A. Aguilar-Arevalo et al. [MiniBooNE Collaboration], arXiv:1002.2680.

[33] A. K. Ichikawa, Lect. Notes Phys. 781 (2009) 17.

[34] A. A. Aguilar-Arevalo et al. [MiniBooNE Collaboration], Phys. Rev. D 81 (2010) 013005.

[35] M. Honda, T. Kajita, K. Kasahara, S. Midorikawa and T. Sanuki, Phys. Rev. D 75 (2007) 043006.

[36] Y. Ashie et al. [Super-Kamiokande Collaboration], Phys. Rev. D 71 (2005) 112005.

[37] D. Rein and L. M. Sehgal, Annals Phys. 133 (1981) 79.

[38] D. Rein, Z. Phys. C 35 (1987) 43.

[39] L. Alvarez-Ruso, S. K. Singh and M. J. Vicente Vacas, Phys. Rev. C 59 (1999) 3386.

[40] T. Leitner, L. Alvarez-Ruso and U. Mosel, Phys. Rev. C 73, 065502 (2006).

[41] J. E. Amaro, E. Hernandez, J. Nieves and M. Valverde, Phys. Rev. D 79, 013002 (2009). 
$\mathrm{K}+$

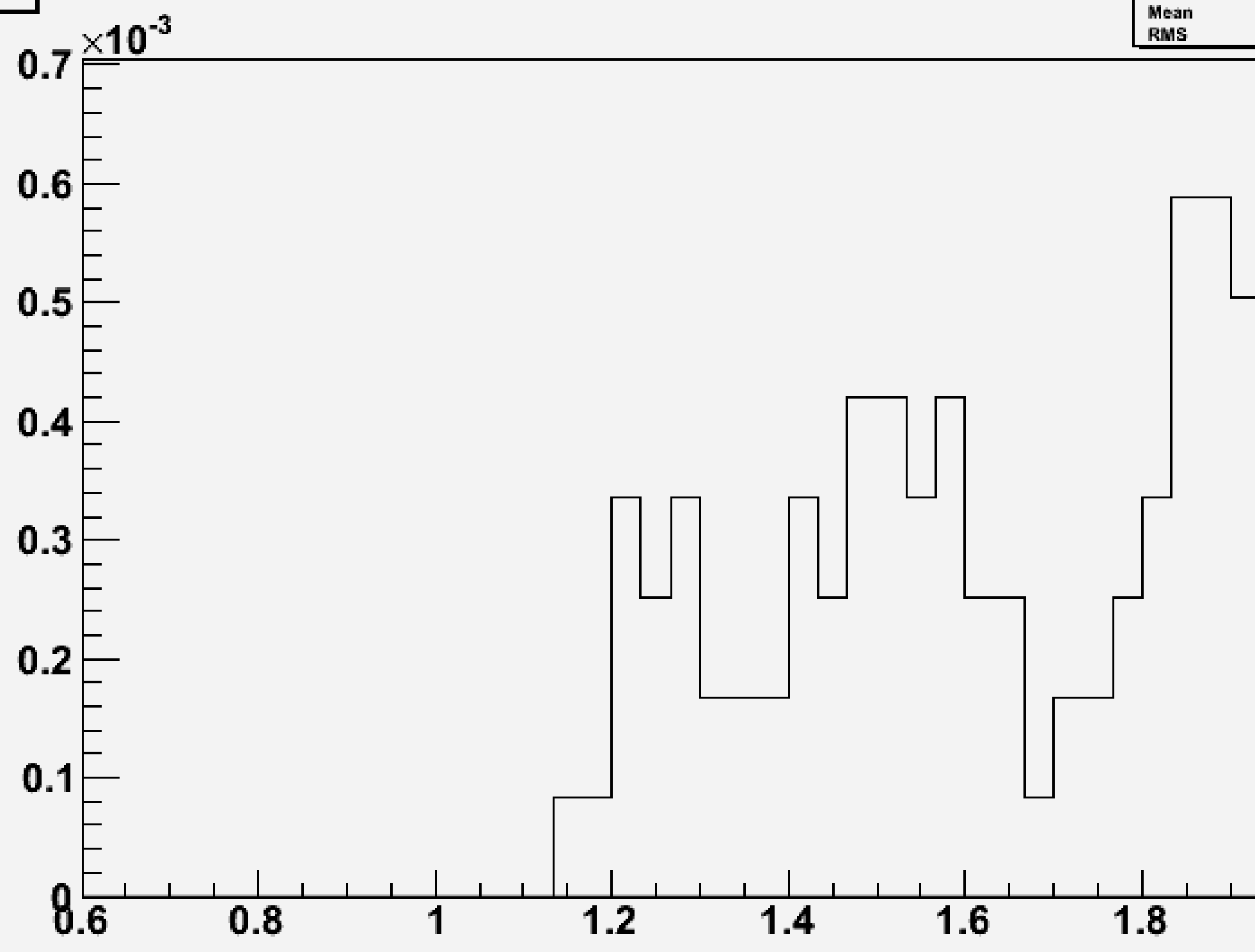

